\title{
UNSTEADY STATE DIFFUSION WITHIN POROUS SOLID PARTICLES
}

\author{
Correlation between Solid Shapes and Diffusion Rates -
}

\author{
SHIGEAKI KASAOKA, YUSAKU SAKATA AND KUNIAKI NITTA** \\ Department of Chemical Engineering, University of Osaka Prefecture, \\ sakai
}

The unsteady state diffusion mechanism has been applied to the analysis or the interpretation for various heterogeneous mass transfer processes such as adsorp$\operatorname{tion}^{12,15)}$, dissolution-extraction ${ }^{4,11)}$, and the intraparticle diffusion controlled reaction ${ }^{5,8}$, for example, ion exchange ${ }^{6,163}$, within porous solid particles. On the other hand, each mathematical solution for unsteady state diffusion and heat conduction within various porous solids; i. e. sphere, infinite cylinder, infinite flat plate, etc. has been obtained by many investigators ${ }^{2,3,7,13,14)}$.

However, as for a fundamental problem, the corre. lation between various solid shapes and intraparticle diffusion or heat conduction rates, the rigorous numerical examination has not yet been established.

In this work, for the purpose of the establishment of the shape correlation, the analytical solutions for the unsteady state diffusion within various shaped solids were derived under the simplified conditions, and then the detailed numerical calculations were carried out.

Furthermore, the approximate estimations for the overall intraparticle diffusion rates within mixed solid particles of different shapes and sizes were examined.

As these results, the correlative values were confirmed to be in good agreements with the analytical values.

\section{Theoretical Equations}

First consider a finite ring porous solid of inner radius $R_{i}$, outer radius $R_{0}$ and length $2 \mathrm{~L}$.

Introducing the intraparticle effective diffusivity, $D_{e}$, and assuming constant shape and constant temperature during the operation, a material balance over a differential volume element within a ring solid yields the following equation in cylindrical coordinates:

$$
\frac{\partial W}{\partial \theta}=D_{e}\left[\frac{1}{r} \frac{\partial}{\partial r}\left(r \frac{\partial W}{\partial r}\right)+\frac{\partial^{2} W}{\partial z^{2}}\right]
$$

An initial condition for the uniform concentration distribution is

$$
W=W_{0} ; R_{i} \leq r \leq R_{0}, \quad-L \leq z \leq L, \quad \theta=0
$$

A boundary condition for the negligible external mass transfer resistance is

$$
W=0 ; r=R_{0}, R_{i}, \quad z= \pm L, \quad \theta>0
$$

The solution of Eq. (1) for these conditions, i. e. the unsteady state intraparticle concentration distribution is

* Received on June 21, 1967

** Ube Kosan Co., Ube determined by multiplying each solution for infinite ring and infinite flat plate as follows.

$$
\begin{aligned}
& \frac{W}{W_{0}}=2 \pi \sum_{m=1}^{\infty} \sum_{n=1}^{\infty} \frac{(-1)^{n-1}}{k_{n}} \cos \left(\frac{z}{L} k_{n}\right) \frac{J_{0}\left(\beta j_{m}\right)}{J_{0}\left(\beta j_{m}\right)+J_{0}\left(j_{m}\right)} \\
& \quad \times\left[j_{0}\left(j_{m} r / R_{0}\right) Y_{0}\left(j_{m}\right)-J_{0}\left(j_{m}\right) Y_{0}\left(j_{m} r / R_{0}\right)\right] \\
& \quad \times \exp \left[-\left(j_{m}{ }^{2}+k_{n}{ }^{2} \gamma^{2}\right)(D \theta)\right]
\end{aligned}
$$

where

$D \theta$ corresponds to Fourier number for mass transfer.

Also, Eq. (4) can be reduced to the solutions for finite cylinder, infinite ring or infinite cylinder by introducing $\beta=0, \gamma=0$ or $\beta=0$ and $\gamma=0$, respectively.

Now, the fraction of a specified material to be transfered out of a porous solid, $f$ is defined as the following expression.

$$
\left.\begin{array}{rl}
f= & 1-\frac{\bar{W}}{W_{0}} \\
\bar{W} & =\frac{1}{V_{p}} \iiint W d V_{p} \\
& =\frac{1}{2 \pi\left(R_{0}{ }^{2} \cdot-R_{i}{ }^{2}\right) L} \int_{R_{i}}^{R_{0}} \int_{-L}^{L} W(2 \pi r \cdot d r \cdot d z)
\end{array}\right\}
$$

Then, by introducing Eq. (4) into Eq. (9), the analytical expression for $f$ is obtained as Eq. (10) shown in Table 1.

The fractions for the other shapes were shown together in Table 1 .

For the systems where a material transfers into a solid from the outer surface, Eqs. (16) and (17) are given as the initial and the boundary conditions instead of Eqs. (2) and (3). Although, of course, each analytical expression for $f$ is given by Eqs. (10) through (15).

$$
W=0 ; \theta=0
$$

$$
W=W_{0} ; \text { on the outer surface, } \theta>0
$$

\section{Numerical Considerations}

Fig. 1 represents curves of the fraction, $f$ against $D \theta$, whose derivatives correspond to the intraparticle diffution rates, according to Eqs. (10) through (15). It is found that these curves spread over the extremely wide range. 
Table 1 Analytical solutions of $f$ against $D \theta$ in a single solid of various shapes

\begin{tabular}{|c|c|c|}
\hline ring & $f_{r}=1-\frac{8}{1-\beta^{2}} \sum_{m=1}^{\infty} \sum_{n=1}^{\infty} \frac{1}{j_{m}{ }^{2} k_{n}{ }^{2}} \cdot \frac{J_{0}\left(\beta j_{m}\right)-J_{0}\left(j_{m}\right)}{J_{0}\left(\beta j_{m}\right)+J_{0}\left(j_{m}\right)} \exp \left[-\left(j_{m}{ }^{2}+k_{n}{ }^{2} \gamma^{2}\right)(D \theta)\right]$ & $\begin{array}{l}J_{0}\left(\beta j_{m}\right) Y_{0}\left(j_{m}\right)=J_{0}\left(j_{m}\right) Y_{0}\left(\beta j_{m}\right) \\
k_{n}=(2 n-1) \pi / 2\end{array}$ \\
\hline cylinder & $f_{e}=1-8 \sum_{m=1}^{\infty} \sum_{n=1}^{\infty} \frac{1}{j_{m}{ }^{2} k_{n}{ }^{2}} \exp \left[-\left(j_{m}{ }^{2}+k_{n}{ }^{2} \gamma^{2}\right)(D \theta)\right]$ & $\begin{array}{l}J_{0}\left(j_{m}\right)=0 \\
k_{n}=(2 n-1) \pi / 2\end{array}$ \\
\hline sphere & $f_{s}=1-6 \sum_{n=1}^{\infty} \frac{1}{k_{n}^{2}} \exp \left[-k_{n}^{2}(D \theta)\right]$ & $k_{n}=n \pi$ \\
\hline $\begin{array}{l}\text { infinite } \\
\text { flat plate }\end{array}$ & $f_{p}=1-2 \sum_{n=1}^{\infty} \frac{1}{k_{n}^{2}} \exp \left[-k_{n}^{2}(D \theta)\right]$ & $k_{n}=(2 n-1) \pi / 2$ \\
\hline $\begin{array}{l}\text { infinite } \\
\text { cylinder }\end{array}$ & $f_{c^{\prime}}=1-4 \sum_{m=1}^{\infty} \frac{1}{j_{m}^{2}} \exp \left[-j_{m}^{2}(D \theta)\right]$ & $J_{0}\left(j_{m}\right)=0$ \\
\hline infinite ring & $f_{r^{\prime}}=1-\frac{4}{1-\beta^{2}} \sum_{m=1}^{\infty}-\frac{1}{j_{m}^{2}} \cdot \frac{j_{0}\left(\beta j_{m}\right)-J_{0}\left(j_{m}\right)}{J_{0}\left(\beta j_{m}\right)+J_{0}\left(j_{m}\right)} \exp \left[-j_{m}^{2}(D \theta)\right]$ & $J_{0}\left(\beta j_{m}\right) Y_{0}\left(j_{m}\right)=J_{0}\left(j_{m}\right) Y_{0}\left(\beta j_{m}\right)$ \\
\hline \multicolumn{3}{|c|}{$\beta=R_{i} / R_{0}, \quad \gamma=R_{0} / L, \quad D=D_{e} / R_{0}^{2}, \quad m, n=1,2,3, \cdots \cdots$} \\
\hline
\end{tabular}

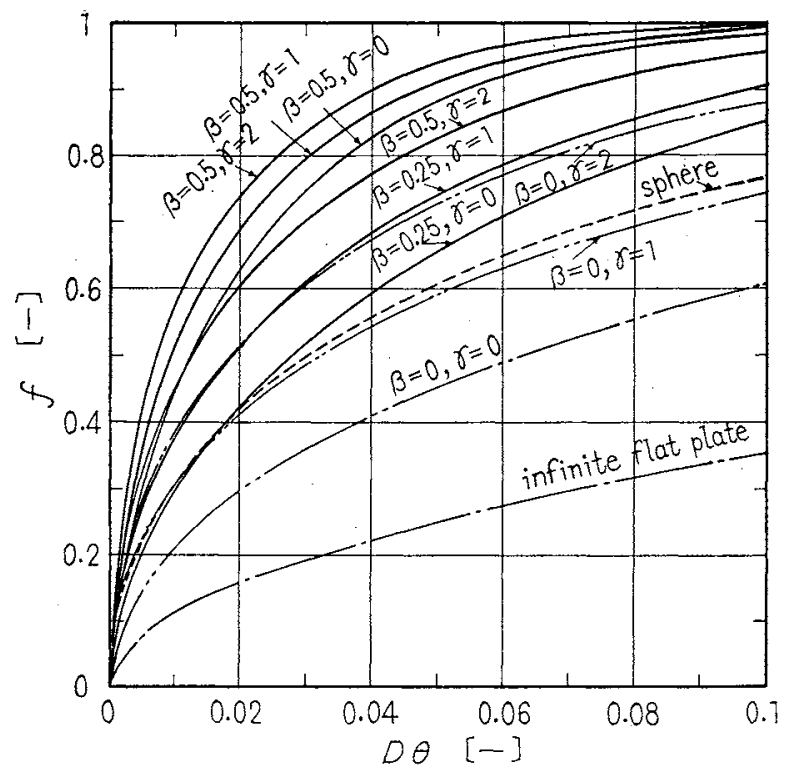

Fig. I Calculation results of Eqs. (10) through (15)

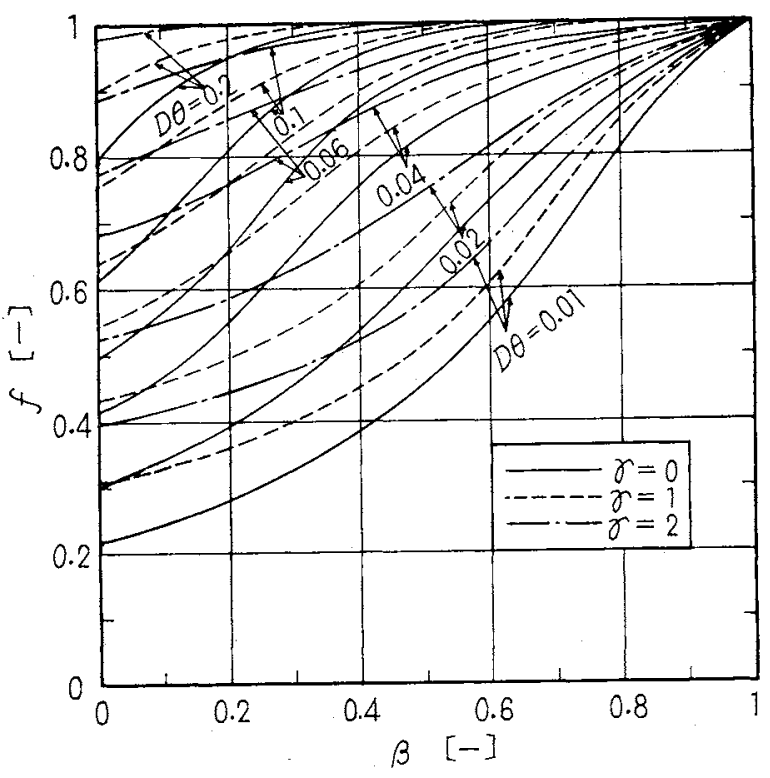

Fig. 2 Effect of $\beta$ on $f$ in a single ring solid; $\beta=0$ corresponds to a cylindrical solid

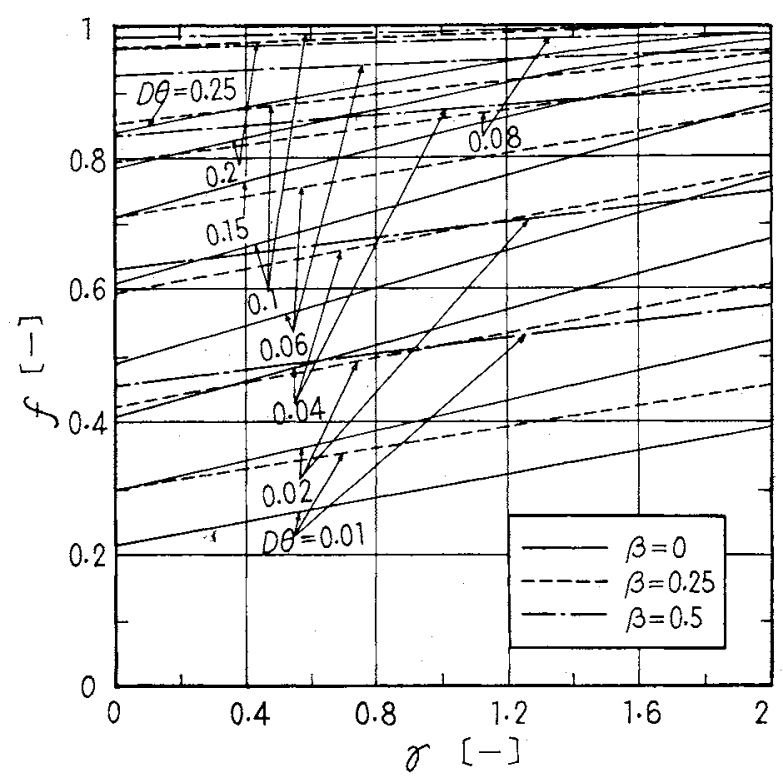

Fig. 3 Effect of $\gamma$ on $f$ in a single ring or cylindrical solid

\section{Effects of $\beta$ and $\gamma$ on $f$ for an Arbitary Ring and Cylinder}

Fig. 2 shows curves of $f$ against $\beta$ at different values of $D \theta$. The variation of $f$ according to the change of $\beta$ can be quantitatively evaluated.

Also, Fig. 3 shows that $f$ varies in approximate proportion to $\gamma$.

The relationships shown in both figures are available for the estimation of $f$ for rings and cylinders of various values of $\beta$ and $\gamma$.

\section{Shape Correlation for an Arbitary Shaped Solid}

As shown in Fig. 1, each curve of $f$ against $D \theta$ is apart far from one another. However, by adopting the ratio, $V_{p} / S_{0}$ as the characteristic dimension of an arbitary shaped solid instead of $R_{0}$ in Eq. (8), i. e. Eq. (18) instead of $\mathrm{Eq}$. (8),

$$
D^{*}=D_{e} /\left(V_{p} / S_{0}\right)^{2}
$$




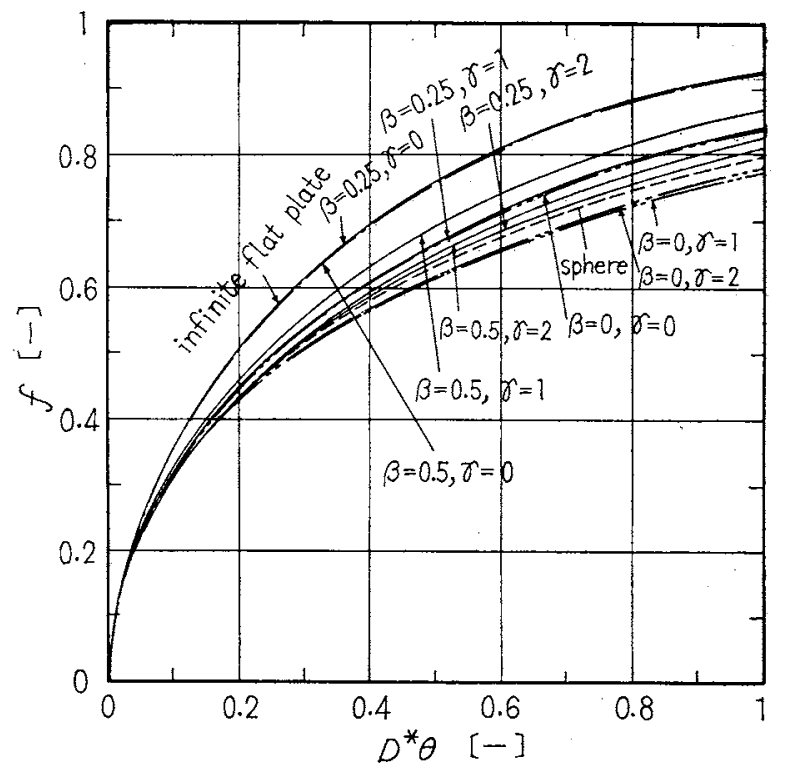

Fig. 4 Calculation results of Eqs. (10) through (15) correlated by Eq. (18) instead of Eq. (8)

these curves approach closely as shown in Fig. 4, although they differ in the strict sense from one another, even if each $V_{p} / S_{0}$ is the same. However, above all, curves for finite cylinders and a curve for spheres coincide with very good accuracy. Also, each analytical value of $f$ for rings can be approximated by that for a sphere, an infinite flat plate or their mean value corresponding to the variation of $\beta$ and $\gamma$.

It was found that the above conclusions are similar to the shape correlation between catalyst particle shapes and the steady state catalytic effectiveness factor ${ }^{1,9,10)}$.

In other words, generally $D \theta$ corresponds to Thiele modulus $\phi_{0}=R_{0} \sqrt{k a_{i} c_{f}^{n-1}} / D_{e}$ in a $n$th order catalytic reaction, and $D^{*} \theta$ to modified modulus $\phi=\left(V_{p} / S_{0}\right)$ $\sqrt{k a_{i} c_{f}^{n-1}} / D_{e}$.

\section{Shape Correlation for a Mixture of Solid Particles of Different Shape-Sizes}

When the unsteady state diffusion rates per unit solid mass or volume are measured with use of the plural solid particles, if the shape or the size of these solid particles is different in every operation, for example, cylindrical solids of various length are employed, the overall rates may be varied in every occasion, and eventually the considerable errors are introduced and the false conclusions may be drawn from the analysis of experimental results, unless the relations among solid shapes employed are quantitatively known.

However, it has not yet established, how to prescribe the characteristic dimension for such mixed systems of solid particles and so the arithmetic mean of particle dimensions appears to be often used. Thus, for obtaining a rational quantitative estimation of the overall rates, the approximations of the overall fraction to be transfered for mixed solids were examined by the numerical calculations.

Now, the general expression of the overall fraction, $f_{0}$ for mixed heterogeneous solids is defined by

$$
\begin{aligned}
f_{0} & =1-\frac{\sum_{j=1}^{a}\left[\left(1 / V_{p}\right) \iiint W d V_{2}\right]_{j}}{\sum_{j=1}^{a}\left(W_{0} V_{p}\right)_{j}} \\
& =\frac{\sum_{j=1}^{a}\left(W_{0} \cdot f \cdot V_{p}\right)_{j}}{\sum_{j=1}^{a}\left(W_{0} \cdot V_{p}\right)_{j}}
\end{aligned}
$$

If both $W_{0 j}$ and $D_{e j}$ for each mixed solid particle are equal, respectively, i. e. if homogeneous solids are mixed, Eq. (19) can be simplified to Eq. (20).

$$
f_{0}=\sum_{j=1}^{a}\left(n_{j} \cdot f_{j}\right) ; n_{j}=V_{p j} / \sum_{j=1}^{a} V_{p j}
$$

Eq. (20) is the definition for mixed homogeneous solid perticles. Accordingly, in order to determine $f_{0}$, it is required that the volume fraction, $n_{j}$ and the fraction, $f_{j}$ for each particle are known. However, the more the number of mixed particles is, the more inconvenient to obtain $n_{j}$ and $f_{j}$ becomes. Thus, the numerical examinations were carried out by the approximate estimation corresponding to Eq. (20).

In this case, the characteristic dimension was remodified as the ratio $\sum_{j=1}^{a} V_{p j} / \sum_{j=1}^{a} S_{0 j}$ and Eq. (21) was adopted instead of Fq. (18).

$$
D_{m}{ }^{*}=D_{e} /\left(\sum_{j=1}^{a} V_{p j} / \sum_{j=1}^{a} S_{0 j}\right)^{2}
$$

Furthermore, for a mixture of ring shape solids, the following $\beta_{m}$ and $\gamma_{m}$ as the overall representative $\beta$ and $\gamma$ were adopted according to Eqs. (21) and (22).

$$
\begin{gathered}
\frac{\sum_{j=1}^{a} V_{p j}}{\sum_{j=1}^{a} S_{0 j}}=\frac{\sum_{j=1}^{a}\left[\alpha_{j}{ }^{3}\left(1-\beta_{j}{ }^{2}\right) / \gamma_{j}\right]}{\sum_{j=1}^{a}\left\{\alpha_{j}{ }^{2}\left(1+\beta_{j}\right)\left[\left(2 / \gamma_{j}\right)+\left(1-\beta_{j}\right)\right]\right\}} \\
=\frac{\left(1-\beta_{m}\right) R_{01}}{2+\left(1-\beta_{m}\right) \gamma_{m}} R_{j=1}^{\sum_{j}^{a}\left[\alpha_{j}{ }^{3}\left(1-\beta_{j}{ }^{2}\right) / \gamma_{j}\right]} \\
\beta_{m}=1-\frac{\sum_{j=1}^{a}\left[\alpha_{j}{ }^{2}\left(1+\beta_{j}\right) / \gamma_{j}\right]}{\sum_{j=1}^{a}\left[\alpha_{j}{ }^{2}\left(1-\beta_{j}{ }^{2}\right)\right]} \\
\sum_{m=1}^{a}\left[\alpha_{j}{ }^{3}\left(1-\beta_{j}{ }^{2}\right) / \gamma_{j}\right]
\end{gathered}
$$

For the information, the followig parameters with the arithmetic mean of each $V_{p} / S_{0}$ were examined.

$$
\begin{gathered}
D_{m}^{*}=D_{e} /\left[(1 / a) \sum_{j=1}^{a}\left(V_{p} / S_{0}\right)_{j}\right]^{2} \\
\frac{1}{a} \sum_{j=1}^{a}\left(V_{p} / S_{0}\right)_{j}=\frac{1}{a} \sum_{j=1}^{a}\left[\frac{\left(1-\beta_{j}\right) \alpha_{j}}{2+\left(1-\beta_{j}\right) \gamma_{j}}\right] R_{01} \\
=\frac{\left(1-\bar{\beta}_{m}\right)}{2+\left(1-\bar{\beta}_{m}\right) \bar{\gamma}_{m}} R_{01} \\
\bar{\beta}_{m}=\sum_{j=1}^{a}\left[\frac{\alpha_{j} \beta_{j}}{2+\left(1-\beta_{j}\right) \gamma_{i}}\right] / \sum_{j=1}^{a}\left[\frac{\alpha_{j}}{2+\left(1-\beta_{j}\right) \gamma_{j}}\right] \\
\bar{\gamma}_{m}=\sum_{j=1}^{a}\left[\frac{2+\left(1-\alpha_{j}\right)+\left(1-\beta_{j}\right) \gamma_{j}}{2+\left(1-\beta_{j}\right) \gamma_{j}}\right] / \\
\sum_{j=1}^{a}\left[\frac{\alpha_{j}\left(1-\beta_{j}\right)}{2+\left(1-\beta_{j}\right) \gamma_{j}}\right]
\end{gathered}
$$


Table 2 Calculation results of Eq. (12) correlated by Eqs. (21) and (26) for a mixture of three through ten spheres of different diameters

\begin{tabular}{|c|c|c|c|c|c|c|c|c|c|c|}
\hline $\begin{array}{c}a \\
{[-]}\end{array}$ & \multicolumn{4}{|c|}{$D^{*} \theta[-]$} & $\begin{array}{c}f_{0}[-] \\
{[\mathrm{Eq} \cdot(20)]}\end{array}$ & $\begin{array}{c}f_{0}\left(D_{m}{ }^{*} \theta\right)[-] \\
{[\mathrm{Eq} \cdot(21)]}\end{array}$ & $\begin{array}{c}f_{0}\left(\bar{D}_{m}^{*} \theta\right)[-] \\
{[\mathrm{Eq} .(26)]}\end{array}$ & $\begin{array}{c}f_{0}\left(D_{m}{ }^{*} \theta\right) / f_{0} \\
{[-]}\end{array}$ & $\begin{array}{c}f_{0}\left(\bar{D}_{m}^{*} \theta\right) / f_{0} \\
{[-]}\end{array}$ & $\begin{array}{c}\left(V_{p} / S_{0}\right)_{\max } / \\
\left(V_{p} / S_{0}\right) \\
{[-]}\end{array}$ \\
\hline 3 & 0.09 & & 0.18 & 0.27 & 0.3571 & 0.3589 & 0.3925 & 1.005 & 1.099 & 1.732 \\
\hline 3 & 0.18 & & 0.27 & 0.36 & 0.4468 & 0.4681 & 0.4841 & 1.003 & 1.037 & 1.414 \\
\hline 3 & 0.27 & & 0.36 & 0.45 & 0.5393 & 0.5402 & 0.5500 & 1.002 & 1.020 & 1.291 \\
\hline 3 & 0.36 & & 0.45 & 0.54 & 0.5950 & 0.5956 & 0.6020 & 1.001 & 1.012 & 1.225 \\
\hline 3 & 0.09 & & 0.18 & 0.9 & 0.3472 & 0.3510 & 0.4354 & 1.011 & 1.254 & 3.162 \\
\hline 4 & 0.18 & 0.27 & 0.36 & 0.45 & 0.4832 & 0.4857 & 0.5104 & 1.005 & 1.056 & 1.581 \\
\hline 4 & 0.18 & 0.27 & 0.36 & 1.8 & 0.4741 & 0.4794 & 0.5555 & 1.011 & 1.172 & 1.897 \\
\hline 5 & $\begin{array}{l}0.045 \\
0.135\end{array}$ & & $\begin{array}{l}0.09 \\
0.162\end{array}$ & 0.108 & 0.2821 & 0.2840 & 0.3149 & 1.007 & 1.116 & 2.236 \\
\hline 5 & $\begin{array}{l}0.18 \\
0.45\end{array}$ & & & 0.36 & 0.4947 & 0.4998 & 0.5488 & 1.010 & 1.109 & 3.162 \\
\hline 8 & $\begin{array}{l}0.09 \\
0.162 \\
0.36\end{array}$ & & $\begin{array}{l}0.108 \\
0.18 \\
0.45\end{array}$ & $\begin{array}{l}0.135 \\
0.27\end{array}$ & 0.3729 & 0.3751 & 0.4154 & 1.006 & 1.114 & 2.236 \\
\hline 10 & $\begin{array}{l}0.045 \\
0.135 \\
0.27\end{array}$ & $\begin{array}{r}c \\
0.36\end{array}$ & $\begin{array}{l}0.09 \\
0.162 \\
\quad 0.45\end{array}$ & $\begin{array}{l}0.108 \\
0.18 \\
0.54\end{array}$ & 0.3100 & 0.3141 & 0.3966 & 1.013 & 1.279 & 3.464 \\
\hline
\end{tabular}

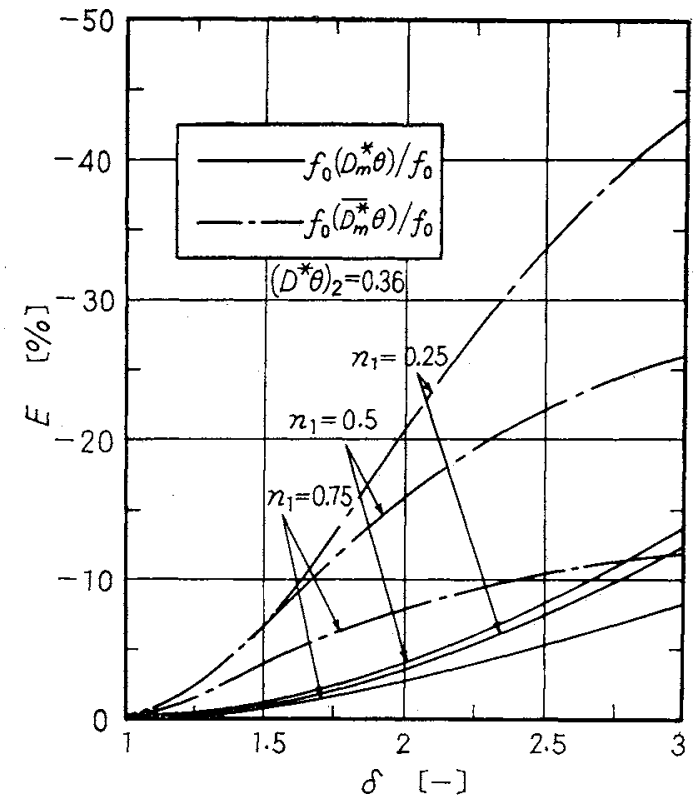

Fig. 5 Error with variation of $\delta$ for a mixture of twa spheres; $\left(D^{*} \theta\right)_{1}>\left(D^{*} \theta\right)_{2}$

For a mixture of cylindrical solids, $\beta_{m}$ and $\bar{\beta}_{m}$ in the above equations become zero.

The results calculated by Eqs. (10) through (12) and Eqs. (20) through (29) indicated great differences between Eq. (21) and Eq. (26), as shown in Table 2 and Figs. 5 through 9.

Also, the overall fraction $f_{0}$ for a mixture of various shapes (for example, spheres, cylinders and rings) of

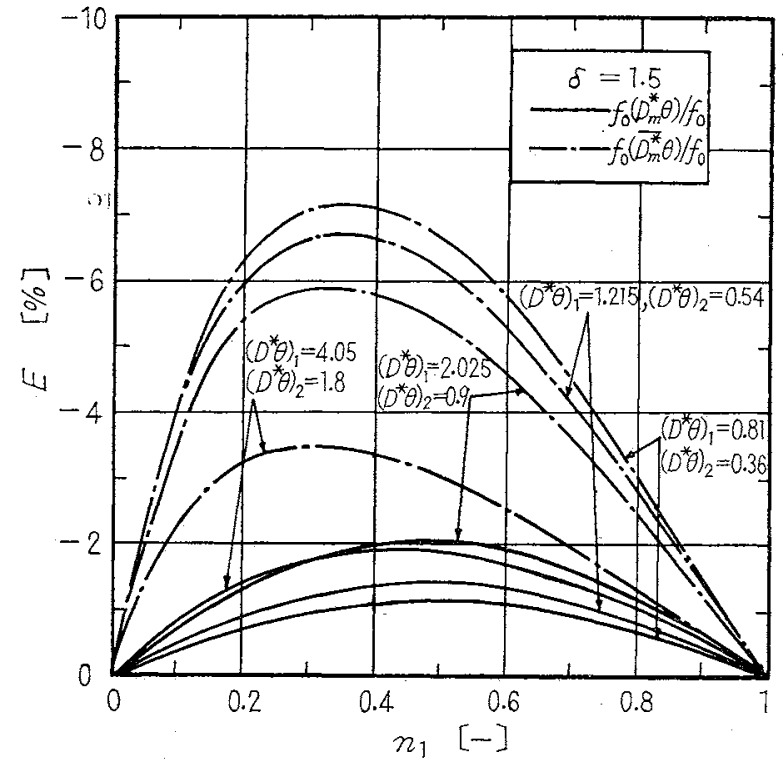

Fig. 6 Error with variation of volume fraction $\left(n_{1}\right)$ and $D^{*} \theta$ for a mixture of many spheres of two kinds diameters

different sizes was approximated by the following equation instead of Eq. (20).

$$
\begin{aligned}
f_{0} \fallingdotseq & f_{0}{ }^{\prime}\left(D_{m}{ }^{*} \theta\right)=\left[n f_{0}\left(D^{*}{ }_{m} \theta\right)\right]_{s}+\left[n f_{0}\left(D_{m}{ }^{*} \theta\right)\right]_{c} \\
& +\left[n f_{0}\left(D_{m}{ }^{*} \theta\right)\right]_{r}
\end{aligned}
$$

where $n$ is the volume fraction of the respective groupes of spheres, cylinders and rings. One of results calculated by Eq. (30) is shown in Table 3 with the correlative values calculated by Eq. (31) etc.. 


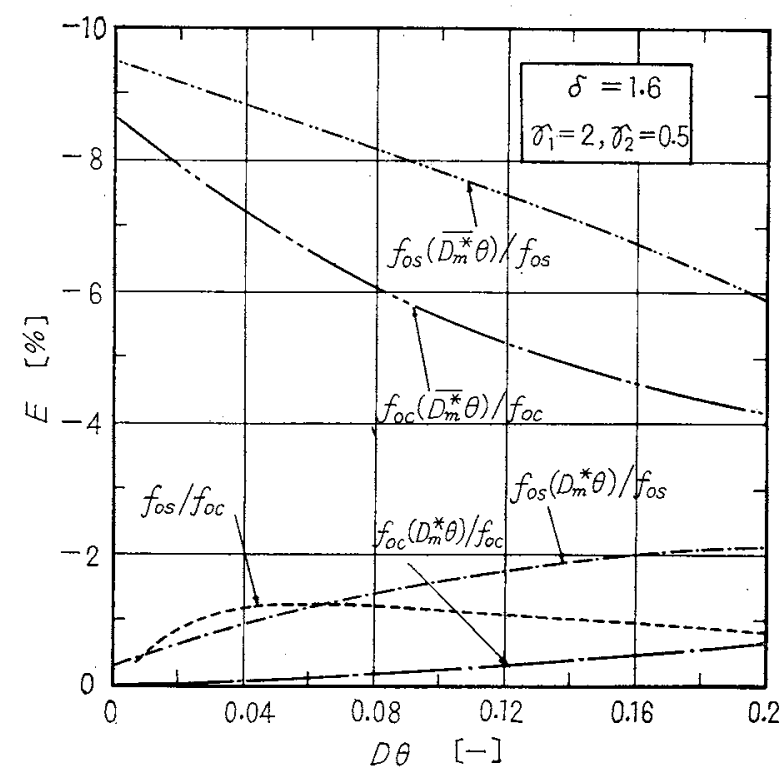

Fig. 7 Error with variation of $D \theta$ for a mixture of two cylinders, and its comparison with correlative value from Eq. (12) for a sphere

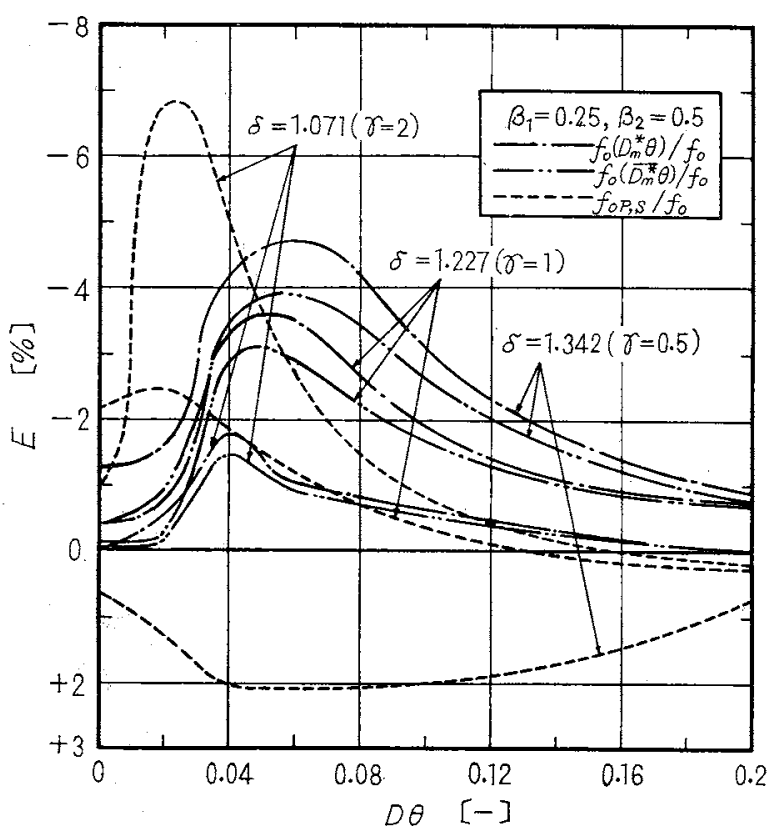

Fig. 9 Error with variation $D \theta$ and $\delta$ for a mixture of two rings of different $\beta$

$f_{0} \fallingdotseq f_{0}^{\prime \prime}\left(D_{m}^{*} \theta\right)=\left[n f_{0}\left(D_{m}^{*} \theta\right)\right]_{s, c}+\left[n f_{0}\left(D_{m}^{*} \theta\right)\right]_{r}$

where $\left[f_{0}\left(D_{m}^{*} \theta\right)\right]_{s, c}$ for spheres and cylinders were calculated by Eq. (12) for spheres, and $\left[f_{0}\left(D_{m}{ }^{*} \theta\right)\right]_{r}$ for rings was obtained as the arithmetic mean value of Eq. (12) for spheres and Eq. (13) for infinite flat plates.

\section{Conclusions}

For the correlation between an arbitrary shape of porous solid particle and the unsteady state intraparticle diffusion rate, $V_{p} / S_{0}$ instead of $R$ as the characteristic dimension can be generally used. Above all, it was

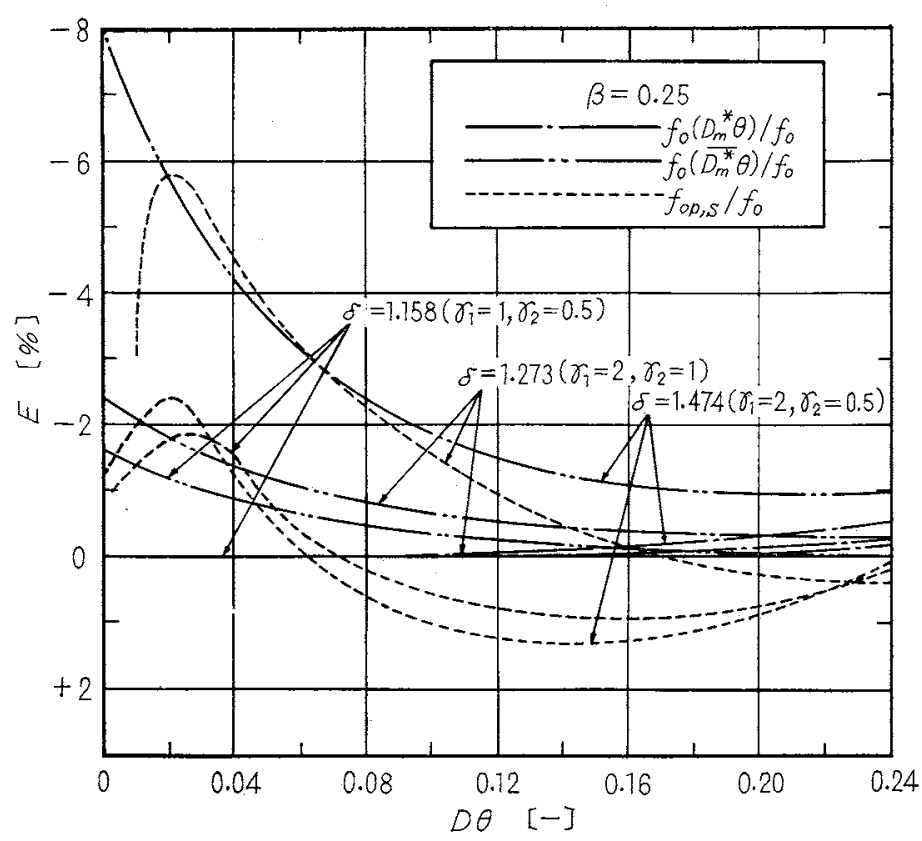

Fig. 8 Error with variation $D \theta$ and $\delta$ for a mixture of two rings of different $r$

confirmed that the analytical values, $f$ for finite cylinders and a sphere coincide with very good accuracy. Also, for a mixture of porous solids of various sizes and shapes, it was found that the analytical values, $f_{0}$ can be estimated with good accuracy by using $\sum V_{p} / \Sigma S_{0}$ instead of $V_{p} / S_{0}$ for a single solid, when the ratios, $\left(V_{p} / S_{0}\right)_{j} /\left(V_{p} / S_{0}\right)_{i}$ are between 1 and about 1.5.

\section{Nomenclature}

a $=$ number of solid particles mixed $[-]$

$a_{i} \quad=$ internal surface area per unit volume of

catalyst particle

$\begin{array}{ll}c_{f} & =\text { concentration of reactant in fluid bulk } \\ & \text { phase }\end{array} \quad\left[\mathrm{mole} / \mathrm{cm}^{3}\right]$

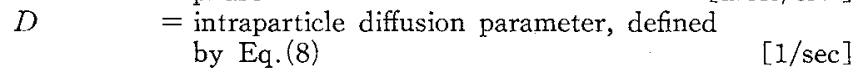

$D_{e} \quad=$ intraparticle effective diffusivity $\quad\left[\mathrm{cm}^{2} / \mathrm{sec}\right]$

$D^{*} \quad=$ modified intraparticle diffusion parameter, defined by Eq.(18) for a single particle [1/sec]

$D_{m}{ }^{*} \quad=$ modified overall intraparticle diffusion parameter, defined by Eq. (21) for a mixture of particles

$\bar{D}_{m}{ }^{*} \quad=$ average intraparticle diffusion parameter, defined by Eq. (26) for a mixture of particles

$E \quad=$ error $=1-$ (correlative value/analytical value) $\times 10^{2}$

$f \quad=$ fraction of a specified material to be transfered, defined by Eqs. (9) etc. for a single particle

$f_{0} \quad=$ overall fraction of a specified material to be transfered, defined by Eq. (20) for a mixture of particles $=f_{0 c}, f_{0 r}, f_{0 s}$ etc.

$f_{0 p, s}=$ overall fraction calculated by the arithmetic mean of Eq. (13) for infinite flat plates, and Eq. (12) for spheres

$f_{0}\left(D_{m}^{*} \theta\right)=$ overall fraction calculated by Eq. (21) and Eqs. in Table 1

$f_{0}\left(\bar{D}_{m}^{*} \theta\right)=$ overall fraction calcultaed Eq. $(26)$ and Eqs. in Table 1

$f_{0}{ }^{\prime}\left(D_{m}{ }^{*} \theta\right)=$ overall fraction calculated by Eqs. in Table 1, Eqs. (21) and (30)

$f_{0}^{\prime}\left(\bar{D}_{m}^{*} \theta\right)=$ overall fraction calculated by Eqs. in Table 1, Eqs. (26) and (30)
$[1 / \mathrm{sec}]$

$[1 / \mathrm{sec}]$

$[-]$

$[-]$

$[-]$

$[-]$ 
Table 3 Calculation results of Eqs. (30) and (31) for a mixture of spheres, eylinders and rings

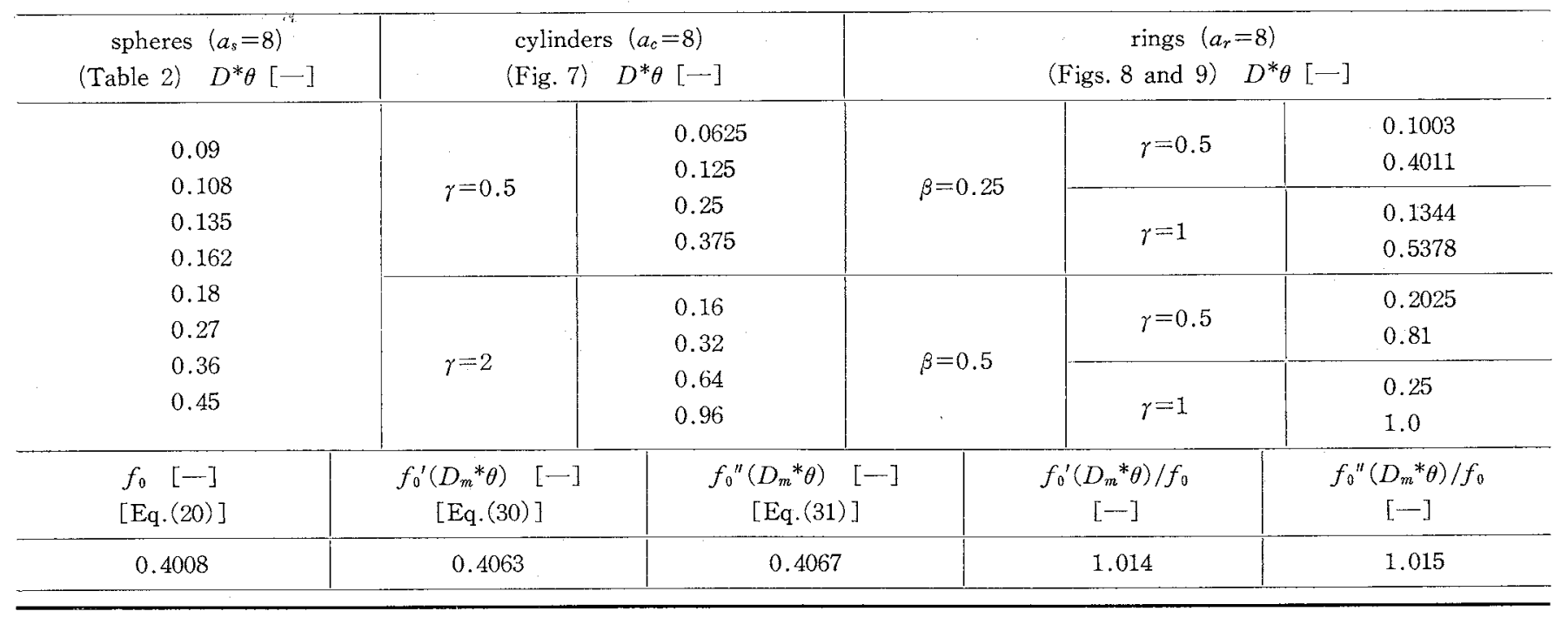

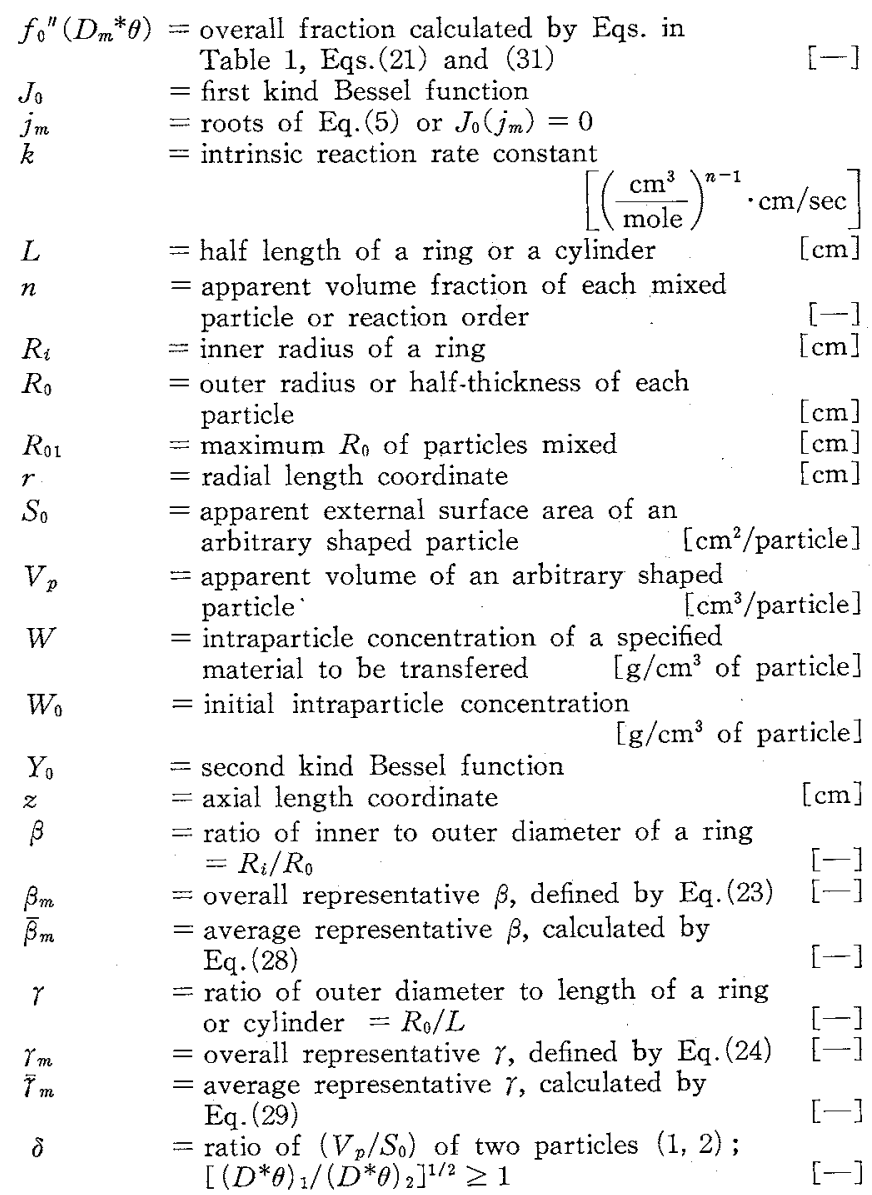

$\theta \quad=$ operation time $\quad[\mathrm{sec}]$

$$
\begin{array}{ll}
\text { subscripts : } & =\text { cylinder } \\
c & =\text { infinite flat plate } \\
p & =\text { ring } \\
r & =\text { sphere }
\end{array}
$$

\section{Literature cited}

1) Aris, R.: Chem. Eng. Sci., 6, 262 (1957)

2) Carslaw, H.S. and J.C. Jaeger: "Conduction of Heat in Solids", 2 nd ed. Oxford, 1959

3) Crank, J.: "The Mathematics of Diffusion", Oxford, 1956

4) Chorny, R. C. and J. H. Krasuk: Ind. Eng. Chem., Proc. Design and Development, 5, 206 (1966)

5) Fujishige, H.: J. Chem. Soc. Japan, Ind. Chem. Sec., 66, 891 (1963)

6) Helfferich, F.: "Ion Exchange", McGraw-Hill, New York, 1962

7) Kamei, S., Y. Urakami and S. Shiomi : J. Chem. Soc. Japan, Ind. Chem. Sec., 40, 804 (1937)

8) Kasaoka, S. and Y. Sakata: Kagaku Kögaku, 30, 50 (1966)

9) Kasaoka, S. and Y. Sakata: ibid., 30, 650 (1966)

10) Kasaoka, S. and Y. Sakata: ibid., 31, 164 (1967)

11) Krasuk, J. H., J. L. Lombardi and C. D. Ostrovsky: Ind. Eng. Chem., Proc. Design and Development, 6, 187 (1967)

12) Masamune, S. and J.M. Smith: A. I. Ch. E. Journal, 11, 34 (1965)

13) McAdams, W. H. : "Heat Transmission", 3 rd ed., McGrawHill, New York, 1954

14) Newman, A. B.: Trans. Am. Inst. Chem. Engs., 27, 203, 310 (1931)

15) Sudo, Y., H. Inoue and S. Yagi: Kagaku Kōgaku, 29, 386 (1965)

16) Turner, J.C. R., M. R. Church, A.S. W. Johnson and C. B, Snowdon: Chem. Eng. Sci., 21, 317 (1966) 